\title{
Phase Diagrams of "Simple" Fluids with Extreme Pair Potentials
}

\author{
C. F. Tejero, ${ }^{1}$ A. Daanoun, ${ }^{2}$ H. N. W. Lekkerkerker, ${ }^{3}$ and M. Baus ${ }^{2}$ \\ ${ }^{1}$ Facultad de Ciencias Físicas, Universidad Complutense de Madrid, E-28040 Madrid, Spain \\ ${ }^{2}$ Faculté des Sciences, CP 231, Université Libre de Bruxelles, B-1050 Brussels, Belgium \\ ${ }^{3}$ Van't Hoff Laboratorium voor Fysische en Colloidchemie, Universiteit Utrecht, Postbus 80051, 3508TB Utrecht, \\ The Netherlands
}

(Received 23 March 1994)

\begin{abstract}
It is shown that strongly reducing the range of the attractions of a simple fluid will transform its phase diagram from the usual type, with a fluid-fluid critical point and a fluid-fluid-solid triple point, into its fluid-solid mirror image, with a solid-solid critical point and a solid-solid-fluid triple point. This scenario could be of relevance to the phase behavior of colloidal dispersions.
\end{abstract}

PACS numbers: $82.70 . \mathrm{Dd}, 81.30 . \mathrm{Dz}$

Colloidal dispersions $[1,2]$ are chemically complex systems whose equilibrium phase behavior can nevertheless be understood in terms of the physical concepts originally coined for the study of simple fluids [3]. Indeed, once the solvent-mediated interactions between the colloidal particles are described in terms of an effective pair potential, the determination of the phase diagram of a complex colloidal dispersion is, in principle, not more difficult than for a simple fluid. If we consider, in particular, a dispersion of sterically stabilized spherical colloidal particles to which nonadsorbing polymer is added, the resulting effective pair potential between the colloidal particles is usually thought to consist of a steep short-ranged repulsion due to particle exclusion and a much weaker attraction due to polymer depletion $[1,2]$. Such a potential is again not unlike that of an ordinary simple fluid like argon [3], except that here the relative range of the attraction versus the range of the repulsion is controlled by the radius of gyration of the added polymer relative to the radius of the colloidal particles and hence can be varied considerably. This then raises the question of general interest of how the phase diagram of a "simple" fluid (including now also the colloids) is modified when the relative range of the attraction versus repulsion is strongly modified. In the present work we address this question by calculating theoretically the phase diagram for a whole family of pair potentials including cases with long-, intermediate-, and short-ranged attractions.

The family of continuous pair potentials, $V(r)$ with $r=|\mathbf{r}|$ the interparticle distance, considered here will be given the following general form, $V(r)=\epsilon \phi(r / \sigma)$ with $x=r / \sigma$ and

$$
\phi(x)=\frac{c}{x}\left(e^{-a(x-1)}-e^{-b(x-1)}\right), a>b,
$$

where $\epsilon$ fixes the temperature scale $\left(k_{B} T / \epsilon\right.$ denoting the reduced temperature $T, k_{B}$ being Boltzmann's constant) while $r=\sigma$ is the zero of $V(r)$ and $\sigma$ will be used to fix the density scale ( $\rho \sigma^{3}$ being the reduced number density $\rho)$. The remaining dimensionless parameters $(a, b, c)$ of (1) are seen to determine the steepness of the repulsion (a), the range of the attraction $(b)$, and the well depth (c). In what follows we will consider a constant well depth, $\phi\left(x_{0}\right)=-1$ with $x_{0}$ denoting the position of the minimum of $\phi(x)$, viz. $\phi^{\prime}\left(x_{0}\right)=0$. This condition will, for given $a$ and $b$, fix $c=c(a, b)$. The family of potentials (1) considered here can thus be parametrized by $(a, b)$ or, equivalently, by the position $x=x_{0}$ of the minimum of $\phi(x)$ and the value $x=x_{1}$ for which $\phi(x)$ has dropped to $1 \%$ of its value at the minimum, viz. $\phi\left(x_{0}\right)=-1$ and $\phi\left(x_{1}\right)=-0.01$ with $x_{1}>x_{0}>1$. Since this is easier to visualize we will henceforth parametrize the potential $\phi(x)$ by $\left(x_{0}, x_{1}\right)$. We will be particularly interested in a sequence of potentials with decreasing $x_{1}-x_{0}$ values, $x_{1}-x_{0}$ being taken here as a measure of the range of the attractions.

To construct the phase diagrams corresponding to (1) we will compute the Helmholtz free energy $(F)$ per particle, $F / N=f(\rho, T)$ with $\rho$ being the number density of the $N$ particles and $T$ the temperature. From $f$ we can obtain the chemical potential $\mu=\partial(\rho f) / \partial \rho$ and the pressure $p=-\partial f / \partial v$, with $v=1 / \rho$. We will consider both fluid and solid phases. For the solid phases we will restrict ourselves to perfect face centered cubic crystals, avoiding hereby any additional complications due to possible structural phase transitions. Below a critical temperature, the free energy of the fluid will, as usual, develop a van der Waals loop with respect to (w.r.t.) $v$ and the fluid-fluid $\left(F_{1}-F_{2}\right)$ coexistences can then be located by performing a double-tangent construction on this loop, since the points of tangency correspond to phases of equal pressure and equal chemical potential. More unexpectedly, we will find that, in complete symmetry with the fluid, the free energy of the solid develops also a van der Waals loop for temperatures below a second critical temperature, corresponding to a much higher critical density. The resulting isostructural solidsolid $\left(S_{1}-S_{2}\right)$ coexistences can again be found by performing a double-tangent construction on the loop of the free energy of the solid. Finally, the solid-fluid $(S-F)$ coexistences are found by performing a double-tangent construction between the free energies of the solid and 
the fluid. Not every coexistence found in this way will correspond to a thermodynamic stable situation. To distinguish the stable and metastable situations one must finally construct, with the help of the free-energy curves and the double tangents, a convex envelope to the free energy. Only those double tangents belonging to this convex envelope will correspond to stable situations.

To obtain the free energy we will use, for both the fluid and the solid, a variational procedure based on the GibbsBogoliubov inequality [3]. This inequality expresses the fact that the free energy is a convex functional of the pair potential and therefore $f$ is always bounded from above by a variational free energy consisting of the free energy of a reference system plus the average, over the reference system, of the difference in energy between the original and the reference system [3]. Our estimate for $f$ will then be given by the minimum, w.r.t. the reference system, of the above variational free energy, for a suitably chosen reference system. The double Yukawa form of (1) is justified here, not so much by the fact that the repulsion between colloidal particles is usually described $[1,2]$ in terms of a Yukawa potential but instead because it leads, for the chosen reference systems, to analytic expressions for the above variational free energy of both the fluid and the solid. This greatly facilitates the large number of minimizations which are required to construct a complete phase diagram. For the fluid phases we have considered a reference system consisting of hard spheres with the hard-sphere diameter as variational parameter. Use of (1) reduces then the computation of the average energy to that of the Laplace transform of $r g(r)$, with $g(r)$ being the hard-sphere paircorrelation function. Both this Laplace transform and the hard-sphere free energy can be obtained in analytic form within the Percus-Yevick approximation [3]. This approach, which was used subsequently, is known to yield a good description of, e.g., the Lennard-Jones fluid [4]. For the reference system of the solid phase we have used an Einstein solid, i.e., a system of identical particles harmonically bound to the lattice sites of the solid, with the common force constant of the oscillators as the variational parameter. The free energy of the Einstein solid is known in analytic form while the use of (1) reduces again the computation of the average energy to an analytic expression having the form of a lattice sum for the Gaussian-averaged potential. This approach is known to yield a good description of, e.g., a solid with a Morse potential [5]. Combining the two approaches we obtain an accurate and simple to implement method which allows us to follow the modifications of the phase diagrams generated by (1) when the parameters $\left(x_{0}, x_{1}\right)$ are modified. We have found three types of phase diagrams which correspond to, say, attractions with a long, intermediate, or short range. These changes seem to be monitored by the value of $x_{1}$, while the position of the minimum $x_{0}$ seems to play only a secondary role. Notice that while $x_{1}$ can be changed freely $\left(x_{1}>1\right)$, the value of $x_{0}$ is always restricted by $x_{1}>x_{0}>1$. We will thus focus our attention mainly on the values of $x_{1}$, which will be considered as a measure of the range of the attraction relative to that of the repulsion. For large $x_{1}$ values $\left(x_{1}>1.6\right)$ we find the usual phase diagram of simple fluids with weak long-range attractions (see Fig. 1). All the $S_{1}-S_{2}$ transitions are metastable, while the $F_{1}-F_{2}$ transitions are stable for temperatures above a $S-F_{1}-F_{2}$ triple point temperature up to the $F_{1}-F_{2}$ critical-point temperature. This type of phase diagram corresponds to a LennardJones-like situation and this can in turn be used here to estimate the accuracy of the above variational method. Indeed, taking $a=14.3959$ and $b=2.6978$, which implies $c=2.0516$, the potential (1) nicely fits the Lennard-Jones
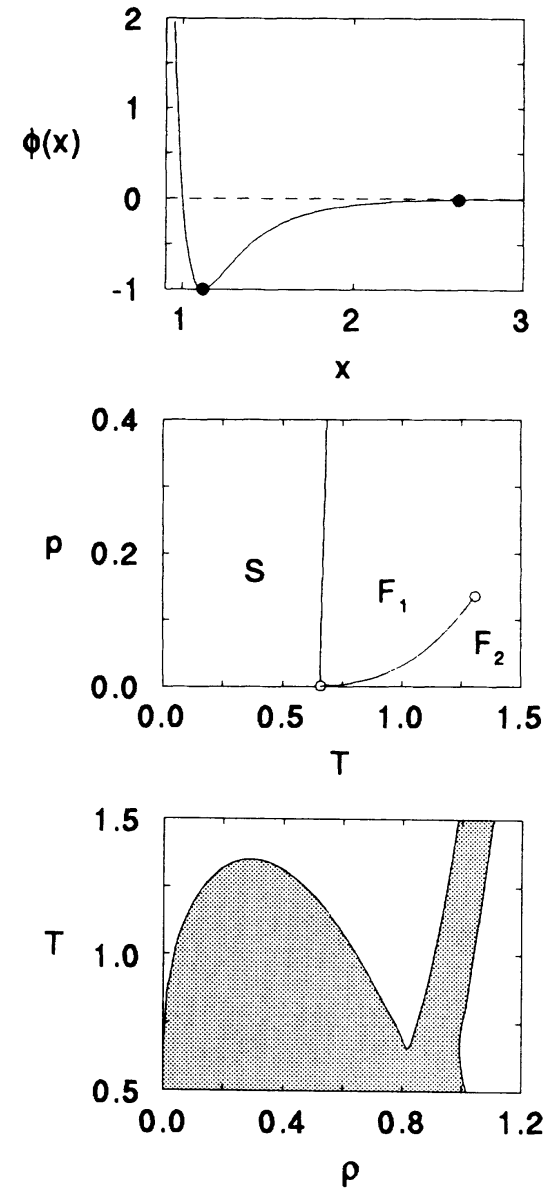

FIG. 1. Phase diagram, in the pressure $(p)$ and temperature $(T)$ and in the temperature $(T)$ and density $(\rho)$ plane (in units such that $\sigma=\epsilon=k_{B}=1$ ) for a simple fluid with the pair potential $\phi(x)$ shown, as obtained from the variational method described in the main text. The case shown here corresponds to a fit of (1) to a Lennard-Jones potential, viz. $x_{1} \simeq 2.62$ and $x_{0} \simeq 1.12$ (full dots). The resulting phase diagram agrees quantitatively with that of the true Lennard-Jones potential [our data for the critical and triple point (open dots) are given in the main text]. This situation is typical for all potentials (1) with long-range attractions $\left(x_{1}>1.60\right)$. 
potential, viz. $\phi_{\mathrm{LJ}}(x)=4\left(x^{-12}-x^{-6}\right)$, because both have $x_{0}=2^{1 / 6} \simeq 1.12$, and, $\phi^{\prime}(1)=-24$, together with $\phi(1)=0, \phi\left(x_{0}\right)=-1$. For this situation $\left(x_{1} \simeq 2.62\right)$ we find for the triple point $T_{t}=0.66, \rho_{t}=0.82$ and for the critical point $T_{c}=1.31, \rho_{c}=0.30$, while the standard results [3] for the Lennard-Jones potential are $T_{t}=0.67, \rho_{t}=0.86$ and $T_{c}=1.36, \rho_{c}=0.36$ in units where $\sigma=\epsilon / k_{B}=1$. For $1.05<x_{1}<1.6$ we find a second type of phase diagram corresponding to what could be termed intermediate-range attractions (see Fig. 2). Here, both the $S_{1}-S_{2}$ and $F_{1}-F_{2}$ coexistences are always metastable and only the $S-F$ transition survives leading to a simple phase diagram without critical or triple points. In going from the previous to the present situation by reducing $x_{1}$ it is seen that the $F_{1}-F_{2}$ criticalpoint temperature is lowered to a value below the $S-F_{1^{-}}$ $F_{2}$ triple-point temperature resulting in a disappearance of the high-density (liquid) fluid phase. This occurs be-
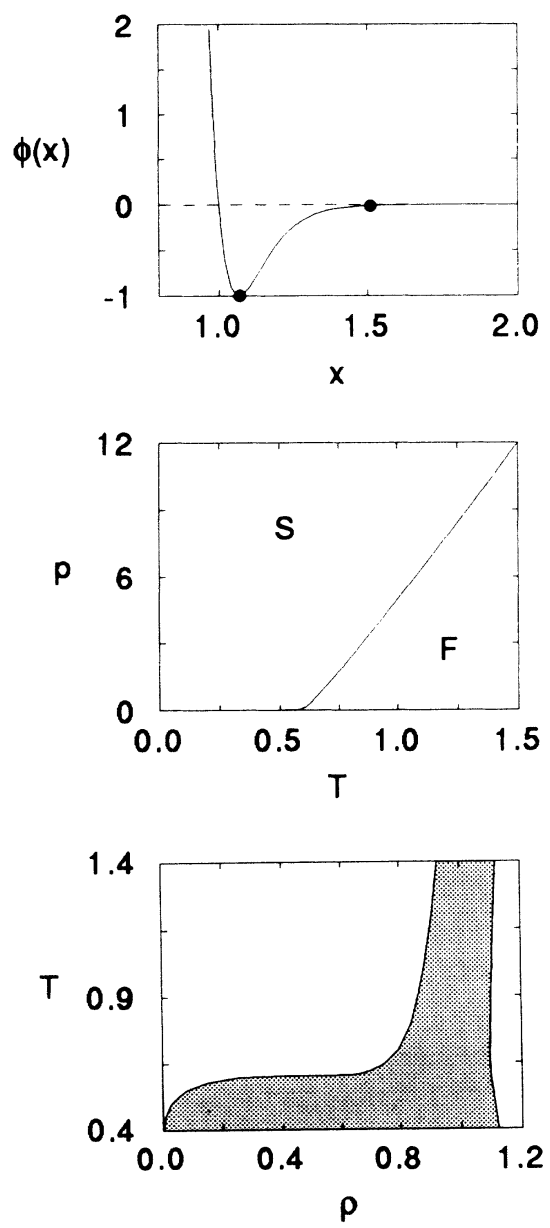

FIG. 2. The same as Fig. 1 but for a potential (1) with $x_{1} \simeq 1.51$ and $x_{0} \simeq 1.07$, i.e., just below the threshold $\left(x_{1} \simeq 1.60\right)$ for the disappearance of the liquid phase. This situation is typical for all potentials (1) with intermediate-range attractions $\left(1.05<x_{1}<1.60\right)$. cause the $F_{1}-F_{2}$ van der Waals loop partly glides above the solid free-energy curve. The threshold value for the liquid to exist appears to be $x_{1} \geq 1.6$, but this value depends still weakly on $x_{0}$. A similar disappearance of the liquid phase has been found in theoretical, experimental, and simulation studies performed for different potentials elsewhere [6-10]. When $x_{1}$ is decreased still further, the $F_{1}-F_{2}$ critical-point temperature continues to decrease while the $S_{1}-S_{2}$ critical point temperature continues to rise which, ultimately, stabilizes the $S_{1}-S_{2}$ transition resulting in a third type of phase diagram (see Fig. 3) with a $S_{1}-S_{2}$ critical point and a $S_{1}-S_{2}-F$ triple point, not unlike the solid-fluid mirror image of the first type. This situation prevails for systems with very shortranged attractions, such that $x_{1}<1.05$, the influence of $x_{0}$ on this second threshold being negligible. For a potential with $x_{1} \simeq 1.019$ and $x_{0} \simeq 1.0025$ we find $T_{c}=2.13$, $\rho_{c}=1.36$ for the $S_{1}-S_{2}$ critical point and $T_{t}=1.84$,
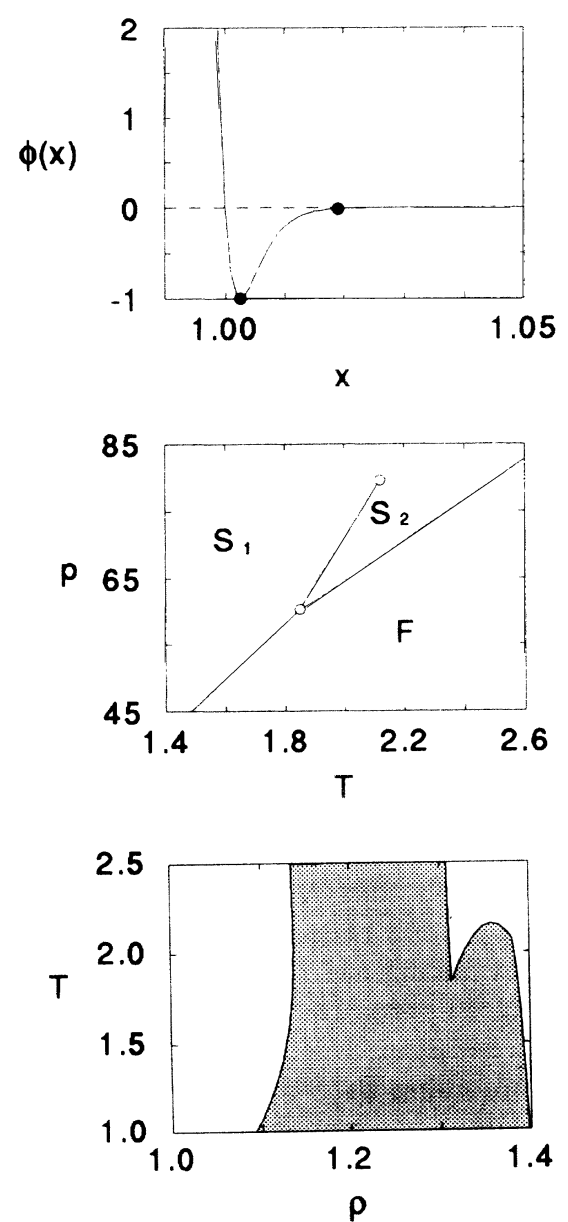

FIG. 3. The same as Fig. 1 but for a potential (1) with $x_{1} \simeq 1.019$ and $x_{0} \simeq 1.0025$, i.e., just below the threshold $\left(x_{1} \simeq 1.05\right)$ for the appearance of an isostructural solid-solid transition. This situation is typical for all potentials (1) with short-range attractions $\left(1<x_{1}<1.05\right)$. This phase diagram is the solid-fluid mirror image of that of Fig. 1. 
$\rho_{t}=1.31$ for the $F-S_{1}-S_{2}$ triple point. An earlier result [11] for adhesive hard spheres did exhibit a van der Waals loop in the solid free energy but the resulting $S_{1^{-}}$ $S_{2}$ transition was unstable. Very recently [12] a similar $S_{1}-S_{2}$ transition was also seen in a computer simulation of hard spheres with a square-well attraction but the fluid phase was not explicitly taken into account in these simulations yielding hereby only a partial phase diagram. Here, instead, we have followed the modifications of the complete phase diagram of a simple fluid described by the continuous pair potential (1) when the range of the attractions is strongly reduced.

In conclusion, a remarkable fluid-solid symmetry of the phase diagram of simple fluids is found when one does allow for potentials with extremely short-ranged attractions. When these attractions are due to depletion forces their range can be experimentally controlled $[1,2]$ and the above scenario could become observable by studying the phase behavior of colloidal dispersions.

C.F.T. wishes to acknowledge the DGICYT (Spain) (PB91-0378). M.B. acknowledges the FNRS (Belgium) and also the Association Euratom-Etat Belge. H.N.W.L. gratefully acknowledges several valuable discussions with D. Frenkel. We thank D. Frenkel for sending us a copy of [12] prior to publication.
[1] W. B. Russel, D. A. Saville, and W. R. Schowalter, Colloidal Dispersions (Cambridge University Press, Cambridge, 1991), 2nd ed.

[2] P. N. Pusey, in Liquids, Freezing and Glass Transition, edited by J. P. Hansen, D. Levesque, and J. Zinn-Justin (North-Holland, Amsterdam, 1991), p. 763.

[3] J. P. Hansen and I. R. McDonald, Theory of Simple Liquids (Academic Press, London, 1976), 1st ed.

[4] B. Firey and N. W. Ashcroft, Phys. Rev. A 15, 2072 (1977).

[5] R. LeSar, R. Najafabadi, and D. J. Srolovitz, Phys. Rev. Lett. 63, 624 (1989).

[6] A. P. Gast, C. K. Hall, and W. B. Russel, J. Colloid. Interface Sci. 96, 251 (1983).

[7] S. Emmett and B. Vicent, Phase Transitions 21, 197 (1990).

[8] H. N. W. Lekkerkerker, W. C-K. Poon, P. N. Pusey, A. Stroobants, and P. B. Warren, Europhys. Lett. 20, 559 (1992).

[9] E. J. Meijer and D. Frenkel, J. Chem. Phys. (to be published).

[10] M. H. J. Hagen, E. J. Meijer, G. C. A. M. Mooij, D. Frenkel, and H. N. W. Lekkerkerker, Nature (London) 365, 425 (1993).

[11] C. F. Tejero and M. Baus, Phys. Rev. E 48, 3793 (1993).

[12] P. Bolhuis and D. Frenkel (to be published). 

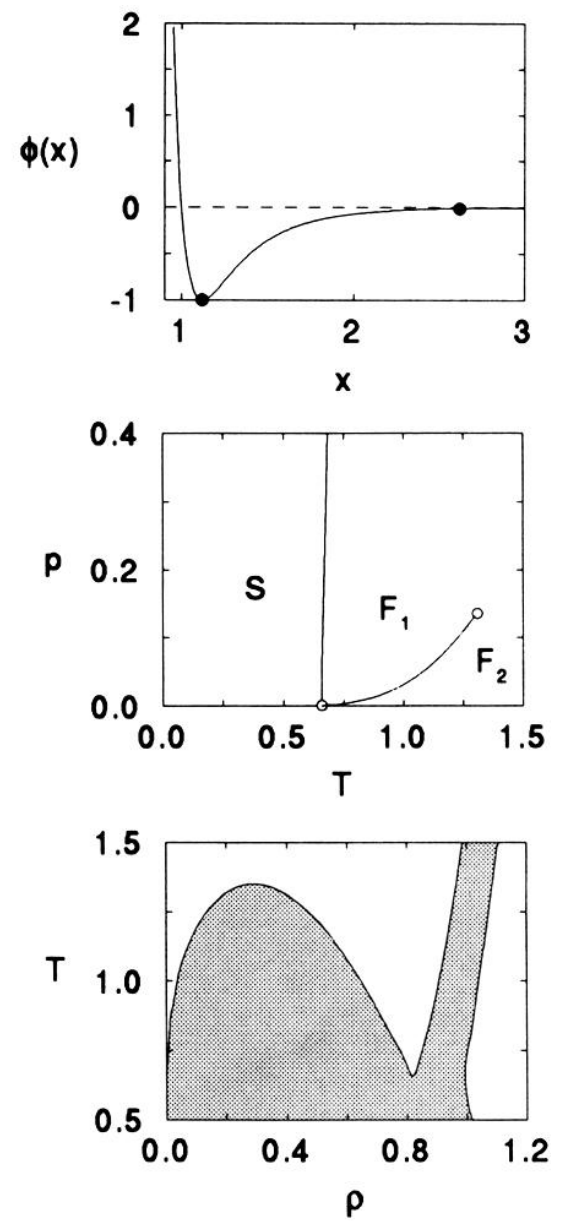

FIG. 1. Phase diagram, in the pressure $(p)$ and temperature $(T)$ and in the temperature $(T)$ and density $(\rho)$ plane (in units such that $\sigma=\epsilon=k_{B}=1$ ) for a simple fluid with the pair potential $\phi(x)$ shown, as obtained from the variational method described in the main text. The case shown here corresponds to a fit of (1) to a Lennard-Jones potential, viz. $x_{1} \simeq 2.62$ and $x_{0} \simeq 1.12$ (full dots). The resulting phase diagram agrees quantitatively with that of the true Lennard-Jones potential [our data for the critical and triple point (open dots) are given in the main text]. This situation is typical for all potentials (1) with long-range attractions $\left(x_{1}>1.60\right)$. 

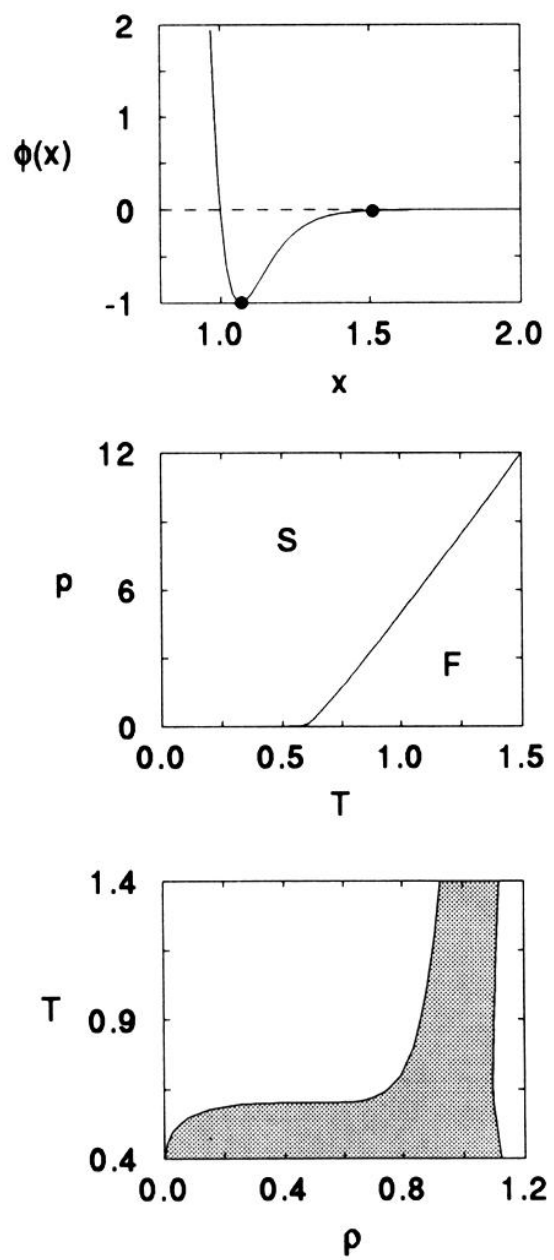

FIG. 2. The same as Fig. 1 but for a potential (1) with $x_{1} \simeq 1.51$ and $x_{0} \simeq 1.07$, i.e., just below the threshold $\left(x_{1} \simeq 1.60\right)$ for the disappearance of the liquid phase. This situation is typical for all potentials (1) with intermediate-range attractions $\left(1.05<x_{1}<1.60\right)$. 

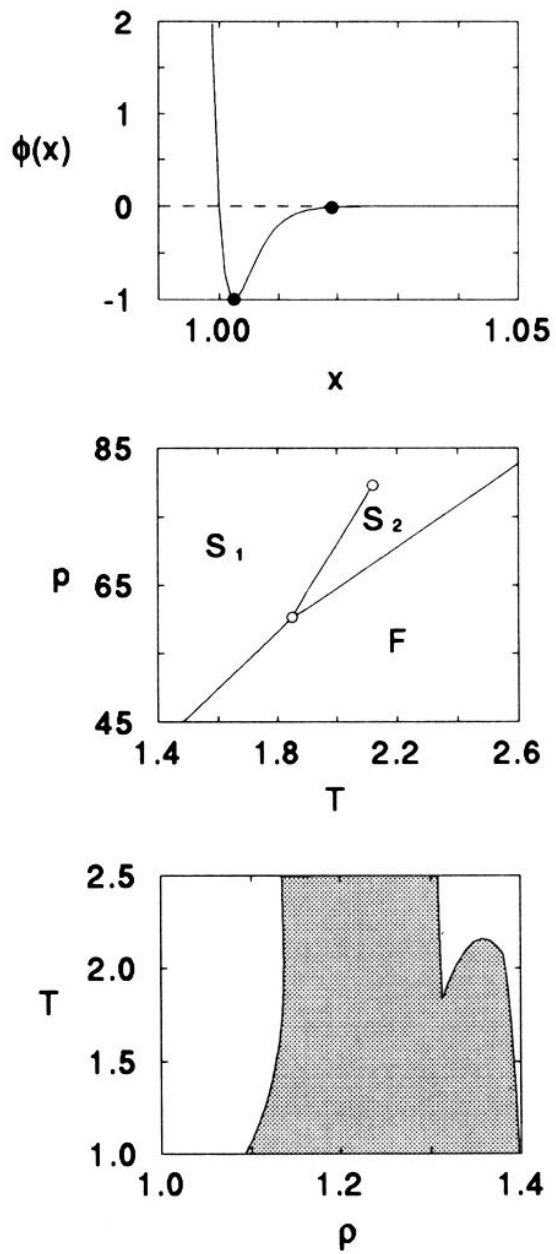

FIG. 3. The same as Fig. 1 but for a potential (1) with $x_{1} \simeq 1.019$ and $x_{0} \simeq 1.0025$, i.e., just below the threshold $\left(x_{1} \simeq 1.05\right)$ for the appearance of an isostructural solid-solid transition. This situation is typical for all potentials (1) with short-range attractions $\left(1<x_{1}<1.05\right)$. This phase diagram is the solid-fluid mirror image of that of Fig. 1. 